\title{
The Theoretical Basis and Feasibility of Time Travel
}

\author{
Meining Du \\ Bachelor of Science, The University of Hong Kong, Hong Kong, 999077, China \\ *Corresponding author:dl_dmn@163.com
}

\begin{abstract}
Whether time travel can be achieved in reality is often a matter of debate, and although many scientists have proposed a number of theoretical ways in which it might be possible, their feasibility remains to be studied. This paper firstly contrasts the absolute spacetime view and relative spacetime view to reflect the understanding of time and other physical quantities in relativity. Subsequently, this paper mainly analyzes seven existing methods of time travel, and introduces them respectively from theoretical principles, related theorems, current development status and development limitations. On this basis, the current situation and prospects of the overall development of time travel are outlined. Besides, the main research method of this paper is to collect various theses related to time travel methods and research data published by some space agencies with integration and analysis as well. This information is sometimes an in-depth study of part of each method, and sometimes a negation of one. But they all help paper to have a more comprehensive understanding, and lead to a conclusion that time travel requires not only the correct theories but also available technology and energy to support the journey to operate and keep astronauts safe.
\end{abstract}

Keywords: Time travel, Relativity, wormhole, Wrap drive, black hole

\section{INTRODUCTION}

Since 1949, when Kurt Godel has found a solution to equations of Einstein in general relativity, which describe a rotating universe, time travel has been widely taken seriously by scientists. The idea of time travel not only reflects desire of knowledge for human, but also influences cosmological view and space-time view for people. Until recently, some of ways in which time travel can be achieved, such as through warp drive and wormholes, have been discovered and some institutions have even begun to try to implement it such as IXS enterprise which is a model produced by National Aeronautics and Space Administration (NASA) and is based on Alcubierre Wrap Drive. Moreover, it is important to note that the abstract term time in the field of relativity and quantum physics is considered to be different from the usual concept in everyday life and Einstein even thinks that time and space are an illusion of human cognition. Due to the variety of methods, it is necessary to organize existing ideas and analyze their limitations and feasibility. This paper mainly explores the research status of time travel and predicts the future development trend by sorting out the views and methods on time and time travel put forward by predecessors as well as browsing a large number of theoretical articles and some technological breakthroughs in real life.

\section{THE BASIC CONCEPT OF TIME AND SPACE AND THE PRELIMINARY EXPLORATION OF TIME TRAVEL}

\subsection{Difference between absolute spacetime view and relative spacetime view}

Time is an abstract physical quantity, and is often used to represent the sequence and continuity of the movement of objects. In view of time and space from Newton which is normally used in daily life, motions in inertial frames of reference do not change space and time, so time and space are absolute. Moreover, there is no necessary connection between time which passes evenly and space which conforms to three-dimensional Euclidean geometry. But in the theory of relativity from Einstein, time and space are not separated but interact with each other to keep the speed of light constant. Here the motion of objects affects space and time, and the time-space coordinates of the different inertial frames are no longer subject to the Galilean transformation but to the Lorentz transformation. The time axis was added to the three-dimensional space, which later evolved into Minkowski four-dimensional spacetime.

For gravity, Newton thought it was the attraction between objects due to mass, but the gravitational law of 
Newton cannot calculate the orbit of Mercury exactly and it is not correct when considering non-static gravitational field [4]. By contrast, Einstein thought that gravity was caused by the distortion of space-time due to mass, and it was a form of space-time. This can fully explain the motion of Mercury.

\subsection{Two inchoate methods of time travel}

While working on solutions to equations from Einstein, Godel guessed that the universe was not expanding and was spinning. Its rotation distorts space to the point where it closes time, so time loops are formed. Time travel is possible when spacecraft travel at slightly less than the speed of light, but this universe does not apply to reality, and the closed loop creates a paradox namely Grandfather paradox.

After rotating universe, Frank Tippler gave another solution to equations from Einstein namely the Tippler Cylinder. It works by twisting a cylinder of infinite length and density around at a very high density and speed and distorting spacetime. Let a spacecraft spiral around a cylinder, leaving it to travel into the past. However, the cylinder involved in this method is difficult to make because it requires a great deal of mass and energy to realize the concept of infinity.

\section{THREE THEORETICAL METHODS OF TIME TRAVEL AND THEIR FEASIBILITY}

\subsection{Wormhole}

Wormhole or Einstein-Lawson bridge was a tunnel connecting two distant space-times when the space-time is curved. It was caused by the gravitational and rotational motion between stars. If the universe is considered as a sheet of paper, then draw a straight line between two arbitrary points on the paper, and this line can be regarded as the shortest distance between two points in the flat universe. When one fold this piece of paper in half and put the pen through two points, then can get a shorter distance than the straight line. The hole one put the pen through can be approximated as a wormhole. Therefore, Wormholes are shortcuts through time.

The concept of wormholes comes from the study of Schwarzschild metric. When studying equations from
Einstein, physicists find that there must be white holes [6]. In addition to the space-time regions of black hole and white hole, there are two isolated space-time regions which can be considered as our universe and its parallel universe. These two universes can both emit particles and signals to the black hole and these things cannot escape from black hole. In the same way, any particles from white hole can emit to the two universes but none of them can return to the white hole. Singularities occur in the regions of black holes and white holes and are represented as hyperbolas. And the point in the middle that connects all four space-times is the Schwarzschild wormhole. This kind of wormhole is one-way due to at one end of the wormhole anything cannot get in, and at the other end anything cannot get out.

Each point in the diagram represents a two-dimensional sphere. I and IV represent two separated universes, with II representing black holes and III representing white holes.

Schwarzschild wormhole is extremely unstable and does not exist long enough for any matter, including light, to travel through them from one universe to the other. Figure 1 shows the evolution of the Schwarzschild wormhole. Two separated black holes and white holes (I) form a wormhole (II). This is followed by instantaneous separation (III) and re-formation of separated black holes and white holes (IV). In that way, matter cannot get in from one end and out from the other, but instead hits the singularity after the wormhole closes. The reason for this phenomenon is that wormholes have very strong gravity, but this strong field of the wormhole can be neutralized by negative energy to stabilize the energy field of the wormhole. This negative energy is provided by negative matter, which not only enables the wormhole to appear more stably, but also ensures that the diameter of the wormhole is large enough, more than 100,000 kilometers, which is the shortest distance for a spacecraft to safely travel. But at the same time, the expanding wormhole will have a "feedback effect" of radiation, similar to the shrill sound generated by a sound loop when a microphone is too close to a speaker. The same goes for radiation, which goes through the loop and amplifies into a significant effect that destroys the wormhole. 


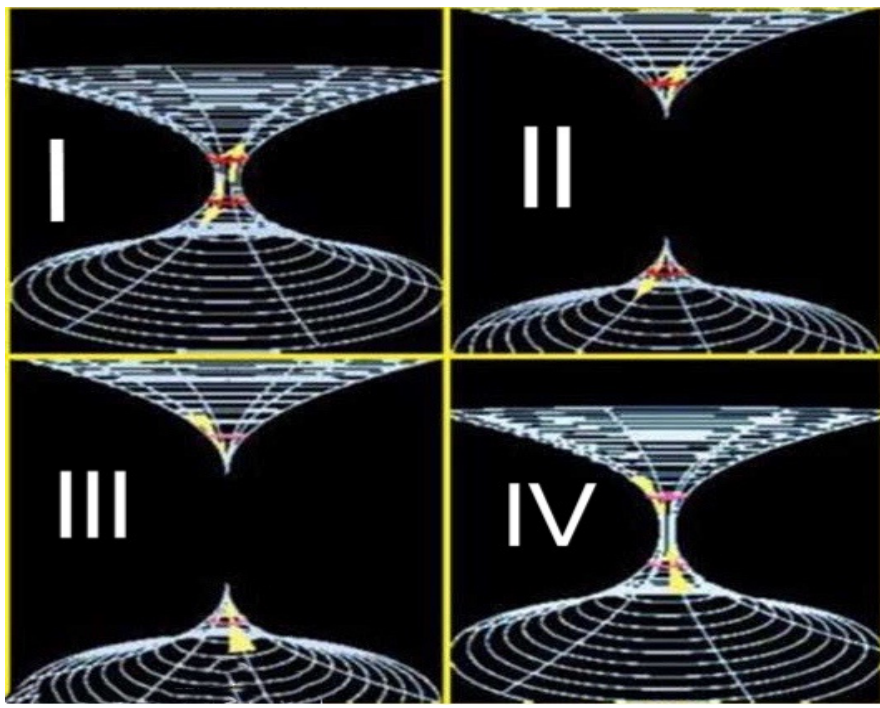

Figure 1. Evolutionary process of the wormhole (From https://www.sohu.com/a/366694539_616747)

As a result, wormhole is only a solution to equations of Einstein, which is just based on mathematical inference. Therefore, it is unknown if there are any shortcuts through space, and wormholes have not been observed. As a result, it remains a mystery whether wormholes can be used for time travel. Stabilizing and expanding the wormhole so that a spacecraft can pass through it will also be a huge challenge.

\subsection{Wrap drive}

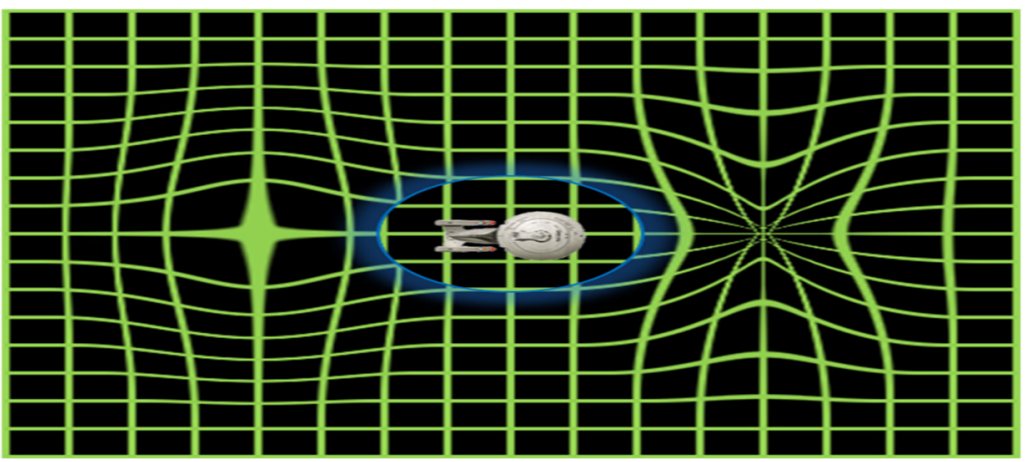

Figure 2. A simulation of how wrap drive changes the surrounding spacetime (From https://tech.ifeng.com/a/20150724/41402604_0.shtml)

However, the safety of wrap drive engines is not so reliable. The one evidence is that the team from Gravity Astrophysics Group at the University of Sydney divided the situation into three types: constant velocity wrap bubbles, one-way wrap bubbles and double-path warp bubbles [2]. It turns out that a wrap bubble with a constant speed would be destroyed and the energy building up in front of the ship, causing it to be destroyed midway or at its destination. A two-way warp bubble is more dangerous than a one-way warp bubble because it has a more destructive flow of high-energy particles. Physicist Miguel Alcubierre of the National Autonomous University of Mexico argues that these
Physicist Miguel Alcubierre proposed warp drive which uses undiscovered substance with negative mass and pressure to drive. This would distort space-time and provide antigravity. As shown in figure 2, the space ship in a wrap bubble, a massive particle in a spacetime distortion, compresses the space in front of it and stretches the space behind it to achieve global superluminal velocity, but at the same time it does not violate the laws of relativity due to local speed is subluminal [3]. Moreover, the wrap bubble is surrounded by flat spacetime, so tidal force and time delays do not affect the work of warp drive [7]. calculations are based on only one dimension, where the situation would not be so terrible [5]. The other thing must be mentioned is that it would produce a lot of radiation that would threaten the lives of astronauts. Last but not least, the astronauts could not send a signal to the front of the wrap bubble, so the spacecraft could not be controlled. The target time for time travel may not be reached. Instead, astronauts may be transported to any time and location. Time travel in this method still have a lot problems to be solved.

In addition to the threat to safety of astronauts, the feasibility of the method is questionable. First of all, there is no evidence for the existence of substance with 
negative mass and pressure. This negative mass will not be attracted by the gravitational field, but will be repelled. Many people believe that negative matter cannot exist because it violates the assumptions of general relativity. But some scientists believe that as long as there is a reasonable way to produce it, it will exist. They thought that both positive and negative mass existed, and that they would form plasma. In this case, no basic assumptions are broken. In addition, some experiments have shown that some substances will behave as negative substances under certain conditions. For example, when the temperature of rubidium atoms drops to near absolute zero, the spin of rubidium atoms can be reversed by using laser traps and exhibit negative mass properties when released. Although these phenomena reflect the characteristics of negative mass, finding such a material with negative mass and energy and even applying it to warp drive engines still requires further research. Furthermore, the realization of the wrap drive requires large amount of negative energy. Specifically, it requires an amount of energy which is similar to the mass of the sun [1]. How this amount of energy can be obtained or how energy needed can be significantly reduced is also extremely important.
Despite limitations of warp drive, many scientists are imagining models or methods to implement it. Mechanical engineer, physicist Harold White from NASA has begun building a prototype spacecraft called the IXS Enterprise. Although it was designed to travel through space, it could also travel through time. As is shown in the figure 3, two large rings around the central spaceship are used to make the Wrap Bubble, which are attached to the main body of the spaceship by sticks. In front of the main body is the place where the operation of the spacecraft is carried out, and behind the main body is the cargo area where the substance with negative mass is stored. Because warp drive was incapable of teleportation, White designed an artificial bubble around the ship that was normal in time and space. He and his partners are looking for ways to reduce the energy required by these bubbles, which they want to fill between the rings. As a result, reasonable mathematical equations are needed for the conversion from concept to feasible design, and in the same way transformation from theoretical model into an actual spacecraft needs methods to use negative energy properly and reduce energy requirements.

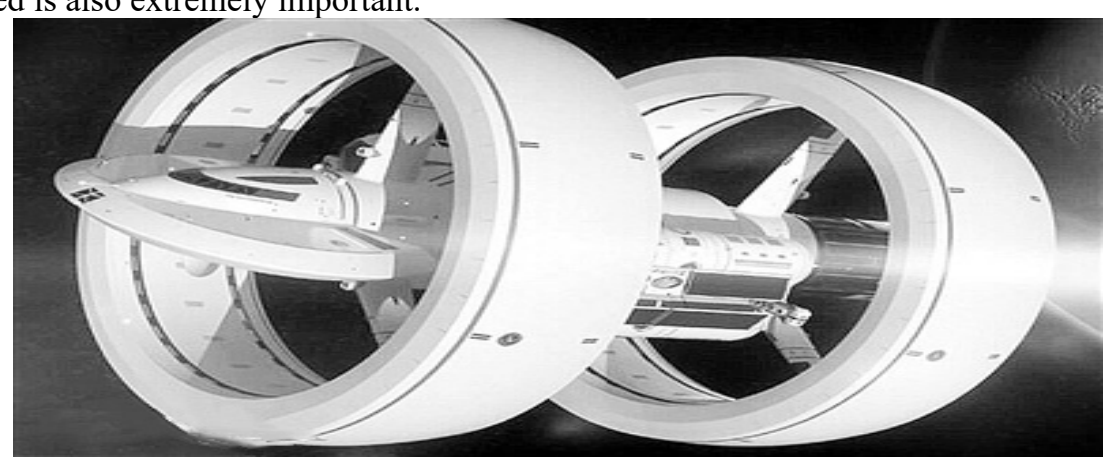

Figure 3. External view of IXS Enterprise (From http://m.haiwainet.cn/middle/345734/2014/0616/content_20746015_1.html)

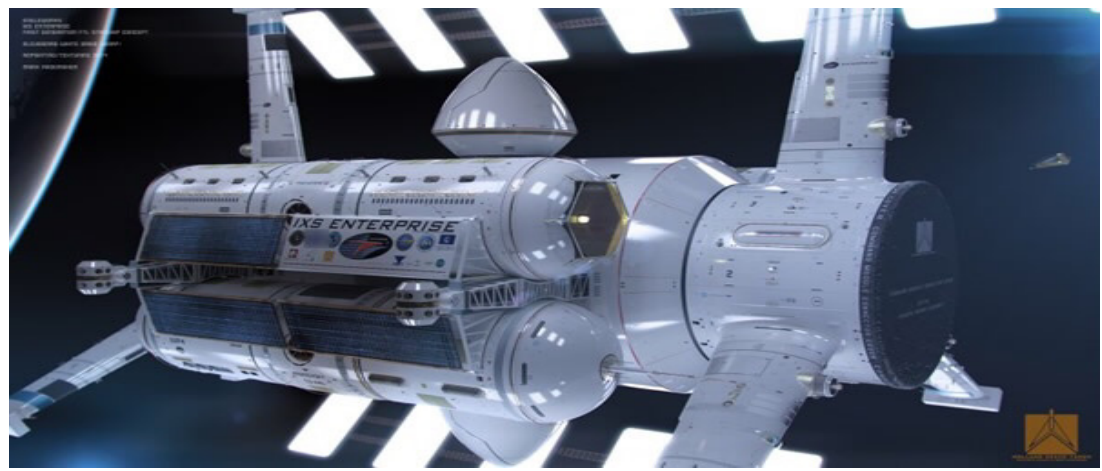

Figure 4. Partly view of IXS Enterprise (From https://tech.huanqiu.com/gallery/9CaKrnQh4Mz)

These two pictures (figure 3 and figure 4) are both model images of the IXS Enterprise, but are presented from different perspectives.

According to the working principle, safety, feasibility and current development of Wrap Drive described above, Wrap Drive is theoretically feasible. However, there is still a long way to go from theory to practice for the search of negative matter, the acquisition of super energy, the maneuverability of spacecraft and the safety of astronauts. But in general, it does count as an important method of time travel, and it has some prospects. 


\subsection{Black hole}

Solution to field equations from Einstein which is calculated by Carl Schwarzschild shows that when the actual radius of a static spherically symmetric star is less than a certain value, the Schwarzschild radius, there is an interface around it called the event horizon. If one enter this interface, not even light can escape. Such objects in the universe are called black holes. The reason light cannot escape from the event horizon is that the gravity of the black hole and the curvature of space-time are so strong that the escape velocity within the event horizon is greater than the speed of light. Unlike wormholes, white holes, and so on, black holes exist and have been photographed. Black holes cannot be directly observed, but their existence is known by $\mathrm{X}$-ray and gamma rays produced by the friction of objects accelerated by gravity before they are sucked into the black hole.

It is necessary to mention here the general relativity understanding of gravity and time. When there is matter, space and time are curved because matter has mass, so gravity is the curvature of spacetime. Large celestial bodies cause the curvature of space-time around them. Small objects enter the curved space-time and move along the curved spacetime. This is definitely a redefinition of gravity, and time is not absolute here. In fact, in places where gravity is strong, such as black holes, time passes more slowly. Here the gravitational red-shift formula can be introduced: $d \tau=\left(1-\frac{2 M}{r}\right) d t . \mathrm{dr}$ is the passage of time felt by an object in a strong gravitational field, called proper time. $d t$ is the time perceived by the person outside the gravitational field, which is coordinate time. It follows that the interval of proper time is smaller than that of coordinate time. The greater the gravity, the greater the curvature of spacetime. As shown in the figure 5, the bending degree of the line segment between two points $\mathrm{A}$ and $\mathrm{B}$ is greater, which can be approximated as $\mathrm{T}=\mathrm{S} / \mathrm{V}$. As the speed of light (V) is constant, the distance traveled (S) increases, and the time schedule in the gravitational field shows a trend of slowing down. Therefore, by the time an astronaut has sailed around a black hole for an hour, it has been a couple of years for people on the ground.

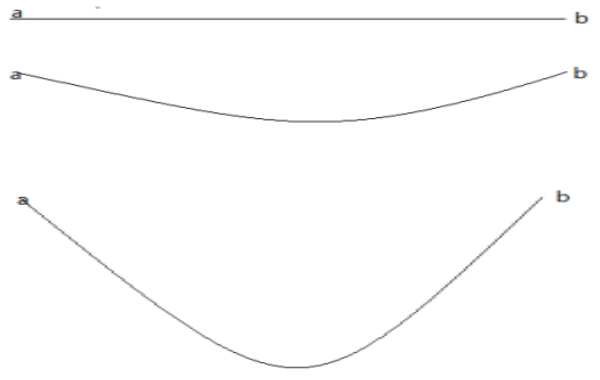

Figure 5. Simulation of spacetime curvature (From https://www.zhihu.com/question/40314696).

However, that is not the kind of time travel people want. It is only possible to travel into the future and only be able to linger around a black hole. In fact, relativity allows people to travel back in time, but it is just a mathematical consequence. Traveling to the past sometimes means the conflict of cause and effect. As Grandfather Paradox pointed out, the existence of ones is against the fact that ones killed their grandfather. However, it makes sense in the multi-world theory that some people actually killed their grandfather in the parallel universe. Therefore, from this perspective, it is possible to travel into the past.

However, all the matter engulfed by the black hole has been broken down into its most basic quantum units, and could be ejected hundreds of millions of years later. From the Quantum DVRS point of view, the black hole does not shatter any information, it just coats it up. It is possible to look at this information if you are just traveling back in time as an outsider. But building a machine that can read information about a black hole will be a challenge.
The nearest black hole is about a thousand light-years away, and it would take us an extremely long time to get there. Whether we could create such a spacecraft is also a question. Furthermore, a black hole has a tremendous gravitational pull and it itself is collapsing enough to tear a man apart, so it would be very difficult to keep an astronaut alive.

\subsection{Other ways to achieve time travel}

\subsubsection{Cosmic string}

The one example is called cosmic string which is one-dimensional and fills the whole universe. Cosmic strings are thought to be long and thin tubes with high energy and large density created during the Big Bang. Due to its high density, it exerts a strong gravitational pull on any nearby object, allowing things on cosmic strings to travel at high speeds. If someone brings two cosmic strings close or one close to a black hole, they will tangle with each other and accelerate, and 
spacetime will be significantly compressed, resulting in closed time-like curves. Hence, the spacecraft around it would be teleported to any time and space. However, the cosmic string is only a theoretical hypothesis and has not been observed because it is so thin. Besides, forming a closed time-like curve requires extremely high energy, which humans have so far been unable to provide.

\subsubsection{Superluminality}

Another method is to travel at a speed which is greater than speed of light. According to Time Dilation and Length Contraction, if an object moves closer to the speed of light, time around it slows down and space shrinks. As a result, some people think that when things move at the speed of light, time stops. Faster than the speed of light, time goes backwards. But according to the theory of relativity, the speed of light cannot be exceeded, so this method is often considered infeasible. If one wants to go faster than light it takes a lot of energy and the Lorentz formula will be imaginary. Therefore, nothing with mass can travel faster than the speed of light.

\section{CONCLUSION}

People have always been curious about time travel and search for the relevant truth, but whether it can be achieved has always been a controversial topic. On the basis of comparing the absolute spacetime view with the relative spacetime view, this paper describes seven theoretical methods of time travel and analyzes their limitations and feasibility. From the discussion in this paper, there are many theories are constantly refreshing our view of spacetime and cosmology. For instance, speed especially near the speed of light and gravity both influence the flow of time and gravity is caused by curvature of spacetime which is due to mass. As a result, the following conclusion can be drawn from the present study is that there are more or less some obvious deficiencies on various kinds of time travel methods. Precisely, some are highly controversial or have been considered impossible, some are not supported by current technology or energy sources, and some are only theoretical and have not been discovered, such as the Wormhole. Although all kinds of limitations are discouraging, these theories and methods give a different understanding of time and space, and each method can cause new thinking and exploration. In addition, it is of great significance to sort out and summarize the methods mentioned in many papers for understanding the general picture of development and further research. However, this paper was limited by detailed and comprehensive development process and the reasoning process of some mathematical formulas. These can be solved by having more detailed and cutting-edge data and materials. Further study can be conducted to have more kinds of ways due to theoretical perfection, or to solve problems in existing methods.

\section{REFERENCES}

[1] B. Alexey, M. Gianni. Introducing physical warp drives. Classical and Quantum Gravity, 2021. doi: 10.1088/1361-6382/abdf6e

[2] B. McMonigal, G.F. Lewis, P. O'Byrne. The Alcubierre Warp Drive: On the Matter of Matter. The University of Sydney, 2012. doi: 10.1103/PhysRevD.85.064024

[3] O.L. Santos-Pereira, E.M.C. Abreu, M.B. Ribeiro. Charged dust solutions for the warp drive spacetime. General Relativity and Gravitation, 2021. doi: 10.1007/S10714-021-02799-Y

[4] F. He. Some studies on entropy of black holes and passage through wormholes. Beijing Normal University, 2002.

[5] M. Alcubierre. The warp drive: hyper-fast travel within general relativity. Class.Quant.Grav, 1994, 73-77. doi: 10.1088/0264-9381/11/5/001

[6] X. Wen, Y. Chen. Wormhole--a tunnel connects space-time. Space Exploration, 2018, 17-19.

[7] Y. Zhou. Negative energy in wrap drive and its related research. Shanghai Normal University, 2014. 